\title{
Highly active antiretroviral treatment against STLV-1 infection combining reverse transcriptase and HDAC inhibitors
}

Philippe V. Afonso ${ }^{1}$, Mourad Mekaouche ${ }^{2}$, Franck Mortreux ${ }^{3}$, Frédéric Toulza ${ }^{4}$, Antoine Moriceau $^{5}$, Eric Wattel ${ }^{3}$, Antoine Gessain ${ }^{1}$, Charles R. M. Bangham ${ }^{4}$, Guy Dubreuil ${ }^{2}$, Yves Plumelle $^{6}$, Olivier Hermine ${ }^{7 *}$, Jérome Estaquier ${ }^{8 *}$, Renaud Mahieux ${ }^{1,9 * q}$

1: Unité d'Epidémiologie et Physiopathologie des Virus Oncogènes, CNRS URA 3015, Institut Pasteur, Paris, France

2: Station primatologie, CNRS, UPS846 D56, Rousset sur Arc, France

3: Unité Oncovirologie et Biotherapies, CNRS FRE3011, Centre Léon Bérard, Lyon, France

4: Department of Immunology, Imperial College, London, United Kingdom

5: Service de pharmacie, Hôpital Necker, Paris, France

6: Service d'hématologie, Centre Hospitalier Universitaire de Fort-de-France, Fort de France, Martinique

7 : Départment d'Hématologie, CNRS UMR 8147, Hôpital Necker, Université Paris Descartes Paris, France

8: INSERM U955, Hôpital Henri Mondor, Créteil, France

9: Oncogenèse Rétrovirale, INSERM U758 / IFR 128 BioSciences Lyon-Gerland / Ecole Normale Supérieure, 69364 Lyon Cedex 07, France.

* OH, JE and RM contributed equally to this work.

II Correspondence to Renaud Mahieux: INSERM U758, Ecole Normale Supérieure de Lyon, 46 allée d'Italie, 69364 Lyon Cedex 07, tel: (33)-4-72-72-88-16, email: renaud.mahieux@ens-lyon.fr 


\begin{abstract}
About three percent of all HTLV-1 infected individuals will develop a disabling inflammatory disease of the central nervous system known as HTLV-1 Associated Myelopathy / Tropical Spastic Paraparesis, against which there is currently no efficient treatment. As correlation exists between the proviral load (PVL) and the clinical status of the carrier, it is believed that diminishing the PVL could prevent later occurrence of the disease. We have conducted a study combining valproate (VPA), an inhibitor of histone deacetylases (HDACi), and AZT, an inhibitor of reverse transcriptase, in a series of baboons naturally infected with STLV-1, whose PVL was equivalent to that of HTLV-1 ACs. We show that the combination of drugs caused a strong decrease in the PVL and prevented the transient rise in PVL that is seen after treatment with HDACi alone. We then demonstrate that the PVL decline was associated with an increase in the STLV-1-specific cytotoxic T-cell population. We conclude that combined treatment with VPA to induce viral expression and AZT to prevent viral propagation is a safe and effective means to decrease PVL in vivo. Such treatments may be useful to reduce the risk of HAM/TSP in ACs with a high PVL.
\end{abstract}




\section{INTRODUCTION}

Ten to 20 million people are infected with human T-Lymphotropic Virus type 1 (HTLV-1) worldwide. This retrovirus is the etiological agent of a malignant lymphoproliferative disease called Adult T-cell Leukemia / Lymphoma (ATLL) ${ }^{1,2}$, and of several inflammatory diseases, particularly of HTLV-1 Associated Myelopathy / Tropical Spastic Paraparesis (HAM/TSP) ${ }^{3-5}$. Despite numerous clinical trials, no efficient treatment has yet been described for HAM/TSP patients. HAM/TSP is a neurodegenerative disease with major irreversible demyelination and loss of motor neurones from the pyramidal tract ${ }^{6-11}$ and the current treatments can only stabilize the clinical status of the patient. It is therefore necessary to develop therapies that can prevent the occurrence of this virus-associated neurodegenerative disease or at least block the evolution of the disease in the early stages of the pathogenesis.

HTLV-1 infects a wide range of cells in vitro. However, in vivo, it is principally detected in $\mathrm{CD}^{+}$T-lymphocytes, and to a lesser extent $\mathrm{CD}^{+}$cells ${ }^{12-16}$. Importantly, there is an association between the HTLV-1 proviral load (PVL) and the clinical status of the individuals. The PVL measured in the peripheral mononuclear cells (PBMCs) isolated from HAM/TSP patients is 5 to 20 -fold higher than that of asymptomatic carriers (ACs) ${ }^{17-21}$. Similarly, HLA genotypes associated with a low PVL are associated with a reduced risk of $\mathrm{HAM} / \mathrm{TSP}^{22,23}$. It is therefore likely that the PVL in an AC will predict the risk of developing an HTLV-1 related disease. In ACs with a high proviral load, diminishing the PVL may also prevent later occurrence of the disease.

Most cells infected with HTLV-1 appear transcriptionally-silent at a given time, which makes therefore viral expression difficult to detect in vivo in ACs even when using highly sensitive techniques such as RT-PCR ${ }^{24,25}$. It is known that the virus replicates mostly, during the chronic phase of infection, via clonal expansion of the infected cells ${ }^{26}$. This pseudo- 
latency renders HTLV-1 resistant to antiretroviral drug treatments in vivo such as AZT, and is likely to play an important role in viral escape from the host immune response ${ }^{24,27}$. As with cellular genes, the expression of the HTLV-1 provirus is epigenetically regulated and the low level of viral expression is associated with proviral chromatin deacetylation and condensation 28,29. A transient transcriptional activation of the viral reservoir should therefore allow a reduction of the PVL in vivo, as long as the host maintains an efficient antiviral immune response.

A recent in vitro study demonstrated that treating HTLV-1 infected lymphocytes with valproate (VPA), an inhibitor of histone deacetylases (HDACi), induces viral expression and cell death ${ }^{30,31}$. A clinical trial with VPA was then initiated in a series of 16 HAM/TSP patients at a late development stage of the disease ${ }^{9}$. This led to a decrease of the PVL after 45 to 60 days of treatment ${ }^{9,32}$. Unfortunately no significant improvement was observed in the clinical status of the patients, perhaps because the HTLV-1-associated neurological damage had already taken place, or because a longer follow-up period was necessary. Moreover, a transient PVL rise was consistently observed in the first weeks of the treatment, probably due to the induction of HTLV-1 proviral expression by VPA and subsequent infectious propagation. Since PVL is linked to disease progression, this observation represents a major concern that currently prevents further use of VPA-based therapy ${ }^{33}$. In this study, the PVL rise was associated with an increase in the 8-meter walking time. It was also pointed out that, in theory, the transient PVL peak might favor CNS invasion (and thus worsen inflammation), as it potentially increases the probability that an HTLV-1 infected lymphocyte will cross the blood-brain barrier ${ }^{32}$. Therefore, preventing the PVL increase at the initiation of treatment should be beneficial in the long term. This trial suggested that VPA might constitute an effective means to prevent or treat HAM/TSP if one could prevent the initial viral replication. 
We therefore conducted a study combining VPA and AZT, an inhibitor of the reverse transcriptase, in a series of baboons (Papio papio) naturally infected with Simian TLymphotropic Virus type 1 (STLV-1). STLV-1 is the simian counterpart of HTLV-1. The two viruses are almost identical at the nucleotide sequence level. Baboons constitute an interesting but little-used model of asymptomatic HTLV-1 infection ${ }^{34}$ : their immune system is very similar to the human, the animals are naturally infected with STLV-1, and some develop STLV-1-associated diseases such as ATLL ${ }^{35}$. We report here that the VPA/AZT combined treatment induced a strong decrease in the PVL in treated animals and prevented the transient rise in PVL observed after treatment with HDACi alone. The PVL decline was correlated with an increase in the STLV-1-specific cytotoxic T-cell population. 


\section{METHODS}

\section{Animal treatment}

Fourteen baboons (Papio papio), naturally infected with STLV-1, were included in this study. Animals were housed at the primate center of the CNRS in Rousset-sur-Arc and cared for in compliance with French regulations. The Regional Committee on Animal Experimentation approved this study. STLV-1 infection was initially determined by serological methods and confirmed by PCR (data not shown). Three animals were left untreated, four animals received VPA alone (Depakine chrono, Sanofi Aventis, France; 500mg/day), two animals received AZT alone (Retrovir, GlaxoSmithKline, UK; 300mg/day) and five received a combination of both drugs (Table 1). The drugs were given per os (embedded in food such as bananas) to each baboon on a daily basis. In all cases, veterinarians ascertained that the drug had been eaten. This was also confirmed by measuring the VPA serum levels for each blood sample, using a routine diagnostic method (data not shown). Treatment toxicity was monitored by behavioral observations, regular weight measurement and transaminase blood level assessment - (aspartate aminotransferase and alanine aminotransferase) (data not shown). The treatment lasted two months. Peripheral venous blood samples from 5 non-infected baboons were also collected in order to compare the initial immune activation level between $\mathrm{STLV}^{+}$and $\mathrm{STLV}^{-}$animals.

PBMCs were purified by Ficoll gradient centrifugation from heparin-anticoagulated peripheral venous blood.

\section{STLV-1 proviral load}

PBMCs were resuspended in lysis buffer (10 mM Tris- $\mathrm{HCl}$ (pH 8.0), 5 mM EDTA, $50 \mathrm{mM}$ $\mathrm{NaCl}, 0.5 \%$ sodium dodecyl sulfate, and $200 \mu \mathrm{g} / \mathrm{mL}$ proteinase $\mathrm{K}$ ) and incubated overnight at $65^{\circ} \mathrm{C}$. DNA was then purified by three consecutive extractions (phenol, phenol/chloroform, 
and chloroform). DNA was precipitated with isopropanol, washed with ethanol, and resuspended in polymerase chain reaction (PCR) grade water. The STLV-1 and albumin specific primers and PCR protocol were previously described ${ }^{36}$. Real time PCR was carried out in a Roche light cycler by using the DyNamo Capillary SYBR Green qPCR kit (Finnzymes, FI) and the Light Cycler Taqman master mix (Roche Diagnostics, IN, USA). Standard curves were generated using serial dilutions of DNA from the MT-4 cell line (which carries 7 proviral copies).

\section{Flow cytometry}

Lymphocytes isolated from heparin-anticoagulated blood were stained with the following monoclonal antibodies: anti-CD3-PerCP (clone SP34-2; BD Biosciences, MA, USA), antiCD4-PerCP or -APC (clone L200; BD Biosciences), anti-CD8-PE or -APC (clone RPA-T8; BD Biosciences), anti-CD20-FITC (clone 2H7, BD biosciences), anti-CD45RA-FITC (clone 5H9; BD biosciences), anti-CD27-RPE (clone M-T271), anti-CD28-FITC or -PE (clone CD2.8, BD biosciences), anti-CD95-PE or -APC (clone DX2, BD biosciences) and anti-Ki67FITC (clone MIB-1; DAKO, DK).

For cell surface staining, antibodies were added to the PBMCs for 1 hour at $4^{\circ} \mathrm{C}$. PBMCs were then fixed and permeabilized with $250 \mu \mathrm{l}$ of Cytofix/Cytoperm (BD Biosciences) for intracellular staining. After washing with Perm\&Wash (BD Biosciences), the cells were incubated for 1 hour with anti-Ki67 antibodies. After washing, the cells were analyzed by flow cytometry as previously described ${ }^{37}$. A total of 20,000 events in the lymphocyte gate were analyzed using FlowJo software (TreeStar, Ashlan, OR, USA).

Absolute numbers of lymphocytes in blood were ascertained using routine diagnostic counts. 


\section{Lytic efficiency assay}

The assay was performed with samples from four animals. The assay quantifies the rate of disappearance of $\mathrm{CD} 4^{+}$Tax-expressing cells caused by addition of autologous $\mathrm{CD} 8^{+}$cells ${ }^{38}$. PBMCs were washed and the $\mathrm{CD} 8^{+}$cells positively selected with anti-CD8 (clone RPA-T8) coupled to magnetic microbeads (Miltenyi Biotec, Germany). The $\mathrm{CD} 8^{+}$cells were then titrated back into the CD8-depleted fraction from the same individual that had been isolated before treatment. Cells were then co-cultivated at $37^{\circ} \mathrm{C}$ for 18 hours, harvested, and stained for Tax ${ }^{39}$, CD4, and CD8 as described above. The proportion of $\operatorname{Tax}^{+} \mathrm{CD} 4^{+}$cells surviving co-culture was plotted against the proportion of $\mathrm{CD}^{+}$cells present. The lytic efficient rate (percentage $\mathrm{CD}^{+} \mathrm{Tax}^{+}$cells killed/\% $/ \mathrm{CD}^{+}$cells/day) was then calculated according to a previously described mathematical model ${ }^{38}$.

The assays were performed in duplicate and the results are presented as the mean CD8+ cell lytic rate ("efficiency").

\section{Statistical analysis}

Analyses were performed in PRISM v.5.0b. We used one-tailed paired Student's t-tests to compare results in paired samples and Mann-Whitney tests to compare unpaired groups. $\mathrm{P}$ values $<0.05$ were considered significant. The non-linear regression presented in figure 5 was determined following a one-phase exponential decay model. 


\section{RESULTS}

\section{STLV-1 infection is associated with changes in T-lymphocyte populations}

We initially compared the immunological profiles of asymptomatic infected $\left(\mathrm{STLV}-1^{+}\right)$and uninfected (STLV-1') baboons. Infected baboons had a higher total number of T-lymphocytes (Mann-Whitney, $\mathrm{p}=0.005$ ) (Figure 1A). However the ratio between $\mathrm{CD}^{+}$and $\mathrm{CD}^{+} \mathrm{T}$-cells remained constant (Figure 1B). The viral infection also decreased the proportion of naïve $\left(\mathrm{CD} 28^{+} \mathrm{CD}^{\circ} 5^{-} \mathrm{CD}^{+}\right.$T-lymphocytes (Figure 1C, $\mathrm{p}=0.02$, Mann-Whitney) and increased the proportion of CD28- (Mann-Whitney, p=0.008) and CD45RA- CD27+ (Mann-Whitney, $\mathrm{p}=0.007) \mathrm{CD}^{+}$effector T-lymphocytes (Figure 1C-D). Similar differences in T-cell phenotypes were reported previously between uninfected humans and HAM/TSP patients ${ }^{40}$. These results support the idea that the immune system of STLV-1 infected baboons is active but unable to clear the infection.

\section{The combination of VPA and AZT reduced the PVL in asymptomatic animals}

We then tested the effect of a treatment consisting of daily doses of VPA alone or in combination with AZT, given per os for 2 months to a series of male and female STLV-1 infected baboons whose PVL was quantified before the initiation of the trial (Table 1). In the absence of treatment, STLV-1 PVL did not change during observation for 2 months (paired Student's t test, $\mathrm{p}=0.27$ ) (Figure 2, upper graph black lines). Consistent with previous HTLV-

1 reports ${ }^{9,41,42}$, AZT had no impact on the PVL after 2 months of treatment (paired Student's t test, $\mathrm{p}=0.11$ ) (Figure 2, upper graph blue lines). As reported in HAM/TSP patients, VPA treatment induced a transient increase in the PVL during the first two weeks in 3 animals out of 4 . However, and contrary to what was previously reported ${ }^{9,32}$, treatment with VPA alone did not cause a significant reduction of the PVL in STLV-1 infected baboons after 2 months 
of treatment (paired Student's t test, p=0.3) (Figure 2, central panel, purple lines). Strikingly, the combined VPA/AZT treatment resulted in a 5- to 12-fold PVL reduction in the 3 animals with a PVL higher than 1 copy per 100 PBMCs. Crucially, the combined treatment prevented the transient increase in mean PVL that is seen after VPA alone (Figure 2, lower graph, orange lines).

\section{Combined treatment with VPA and AZT resulted in an accumulation of CD8+ lymphocytes}

Because the increase in PVL occurred in animals treated with VPA alone during the first week of treatment, we focused our immunological analyses on this period. There was no significant variation in the absolute number of T-cells either in control (untreated) or AZTtreated animals (Figure 3A, upper row) during the first week of the trial (paired Student's ttest, $\mathrm{p}=0.9$ and $\mathrm{p}=0.8$ respectively). In all but one VPA-treated animals, the total T-cell number decreased especially for $\mathrm{CD}^{+} \mathrm{T}$ cells (paired Student's t-test, $\mathrm{p}=0.045$ ) (Figure 3A, central row). Interestingly, animal V2, which had no transient increase in PVL, also displayed an increased total lymphocyte number. Conversely, in the VPA-AZT treated animals, the decrease in PVL was associated with a significant increase in the number of lymphocytes (paired Student's t-test, $\mathrm{p}=0.016$ ), principally of the $\mathrm{CD} 8^{+} \mathrm{T}$ cell population (Figure 3A, lower row). In the 2 animals that did not display a major change in PVL following VPA/AZT treatment, the total lymphocyte count also remained approximately constant.

\section{Combined VPA/AZT treatment results in an accumulation of CD8+ effector lymphocytes}

In order to determine which T-cell subpopulations were altered during the treatment, naïve, central memory and effector $\mathrm{CD} 8^{+}$cell populations were analyzed one week post-initiation of 
the treatment using two sets of markers: CD28/CD95 (figure 3B) and CD45RA/CD27 (Supplemental Figure 1). The numbers of $\mathrm{CD} 28^{+} \mathrm{CD} 95^{-}$(naïve) and $\mathrm{CD} 28^{+} \mathrm{CD}^{+} 5^{+}$(central memory) $\mathrm{CD}^{+} \mathrm{T}$-cells were unchanged in all four groups of animals (Kruskal-Wallis, $\mathrm{p}=0.43$ and 0.62 respectively). However, significant changes were observed in the $\mathrm{CD} 8^{+} \mathrm{CD} 28^{-}$ (effector) T-cell population (Figure 3B, right panels). The animals treated with VPA had a decrease in the number of $\mathrm{CD} 8^{+} \mathrm{CD} 28$ - (effector) $\mathrm{T}$ cells (paired Student's t-test, $\mathrm{p}=0.039$ ), with the exception of the animal V2. In the VPA/AZT-treated animals, whose PVL decreased upon treatment, the number of effector $\mathrm{CD} 8^{+} \mathrm{CD} 28$ - T cells increased (paired Student's t-test, $\mathrm{p}=0.039)$. This result was confirmed with the second set of differentiation markers: the CD8 ${ }^{+}$ $\mathrm{T}$ cell population whose number fluctuated was mainly $\mathrm{CD}^{4} 5 \mathrm{RACD}^{-}{ }^{-} 7^{-}$(effector memory) (paired-Student's t-test, $\mathrm{p}=0.02$ and 0.036 for VPA and VPA/AZT respectively) (Supplemental figure 1). In contrast, the $\mathrm{CD} 4^{+} \mathrm{T}$-cell count was not altered (Supplemental Figure 2).

To determine the origin of the $\mathrm{CD}^{+}$population expansion, lymphocyte proliferation was quantified by analysis of Ki67 expression (Figure 4). The proportion of proliferating CD4 ${ }^{+}$ cells was not modified in untreated, AZT-treated or VPA-treated monkeys (data not show), although a rebound in viral replication was observed in this latter group at 1 week of treatment. This observation strongly suggests that the transient PVL increase observed during VPA treatment is the consequence of new cycles of infection within the PBMCs. Conversely, the accumulation of effector $\mathrm{CD}^{+} \mathrm{T}^{-1 y m p h o c y t e s}\left(\mathrm{CD} 28^{-}\right)$in VPA/AZT-treated animals was associated with an increase in proliferation of the $\mathrm{CD}^{+}$central memory $\mathrm{T}$-cell $\left(\mathrm{CD} 28^{+} \mathrm{CD}^{+} 5^{+}\right)$subset (paired Student's t-test, $\left.\mathrm{p}=0.035\right)$ (Figure 4, central panel), consistent with the normal pathway of T-cell differentiation. 
STLV-1 PVL reduction correlated with an accumulation of anti-retroviral cytotoxic lymphocytes

We determined whether the accumulation of effector $\mathrm{CD}^{+} \mathrm{T}$-cells in VPA/AZT treated monkeys represented an increase in the population of cytotoxic lymphocytes (CTL) directed against STLV-1. To this end, we determined the rate of $\mathrm{CD}^{+}$-mediated lysis of autologous, naturally STLV-1-infected cells at different time points (days 0, 7 and 15 of treatment). The results revealed a correlation between the variation in the CD8+ cell-mediated lysis rate and the variation in STLV-1 PVL (Figure 5). 


\section{DISCUSSION}

We analyzed the impact of a combination of valproate (VPA), an inhibitor of histone deacetylases (HDACi), and AZT, an inhibitor of the reverse transcriptase, in asymptomatic baboons naturally infected with Simian T-Lymphotropic Virus type 1 (STLV-1).

These non-human primates constitute the best model for studying HTLV-1 associated diseases and potential therapies, since baboons are the natural hosts for STLV-1, a retrovirus closely related to HTLV-1. In simians, as in humans, the infection remains mainly asymptomatic. However, cases of ATLL-like retrovirus-associated diseases have been reported ${ }^{35}$. We show here that the differences in the T-lymphocyte subpopulations associated with STLV-1 infection in baboons are also similar to those described in HTLV-1-infected humans ${ }^{40}$. In each case, the retroviral infection is associated with an accumulation of effector T-lymphocytes, which represent a marker of an immune response against the virus. One means of retroviral escape from the immune response is proviral silencing through epigenetic regulation. This is the reason why we hypothesized that drug-induced viral activation would promote viral clearance by the immune system.

A previous study described the use of VPA in HAM/TSP patients, which resulted in a significant decrease in the PVL after 45-60 days of treatment ${ }^{9}$. We therefore hypothesized that VPA-based treatments could be given to ACs at risk of developing an HTLV-1 associated disease. Surprisingly, in our assay, administration of VPA alone did not cause any reduction in PVL during 2 months of treatment. However, and consistent with the previous report ${ }^{9}$, we observe a transient but significant (up to a 3-fold increase) rise in PVL during the first week of treatment, suggesting that VPA treatment is not only unsuccessful, but might be hazardous by increasing the rate at which HTLV-1-infected lymphocytes cross the blood-brain barrier, as suggested previously ${ }^{32}$. 
In contrast, the VPA/AZT combination prevented the transient rise in PVL caused by VPA alone. More interestingly, it also induced a significant reduction in PVL in animals with a high initial PVL. The decrease in PVL was associated with an accumulation of effector CD8+ T-lymphocytes and cytolytic lymphocytes directed against the virus. This strongly suggests that the observed PVL decrease was not due to a direct apoptotic effect of the VPA on infected cells, as suggested by in vitro studies ${ }^{30,31}$, but rather to an increase in the effectiveness of the anti-STLV-1 cellular immune response. The accumulation of CTL is a consequence of an increased proliferation of memory CD8+ T-cells. The proliferation of memory cells and subsequent accumulation of effector cells was not seen in the VPA group. We therefore suggest that the observed reduction in PVL was caused by an efficient immune response against the virus when de novo infection was prevented by addition of AZT. Our current working model is the following: in VPA/AZT treated animals, infected cells express the virus in response to VPA treatment. When central memory lymphocytes recognize these cells, they proliferate, differentiate into effector cells, and kill STLV-1 infected cells, which leads to a decrease in PVL. On the contrary, in the absence of AZT, the virus spreads, STLV1-specific central memory cells become infected, and are destroyed by autologous CD8+ T cells in a 'fratricidal' response ${ }^{13}$. Thus, the number of proliferating virus-specific memory cells decreases, and cannot reconstitute the effector $\mathrm{CD} 8^{+}$reservoir. As a result, the PVL may increase.

As stated above, the proviral load is the strongest known correlate of inflammatory diseases in HTLV-1 infection. However, the only described therapy that reduces the PVL causes a large initial transient rise before the eventual fall ${ }^{9}$. Our present data represent therefore the first indication that it is possible to make a substantial reduction in the PVL of an oncogenic retrovirus without a concomitant significant rise in the PVL shortly after starting treatment. This suggests that, when biomarkers are available, VPA/AZT could be 
given to HAM/TSP patients at the early stage of the disease. Finally, a recent nationwide prospective study conducted in Japan strongly suggested that a higher PVL is a strong factor in the development of ATLL ${ }^{43}$. The VPA/AZT combination may therefore be used as a preventive treatment for other HTLV-1 related diseases such as ATLL. 


\section{ACKNOWLEDGMENTS}

We gratefully thank Sylviane Bassot for the serological analyzes and Emilie Coté for helpful suggestions. We also acknowledge the RsA-FD for their support.

\section{AUTHORSHIP}

PVA and RM designed research, performed research, analyzed data and wrote paper; MM FM FT AM FM performed research; EW CB YP analyzed data; AG JE OH GD designed research.

Conflict-of-interest disclosure: The authors declare no competing financial interests.

* $\mathrm{OH}, \mathrm{JE}$ and RM contributed equally to this work.

II Correspondence to Renaud Mahieux: INSERM U758, Ecole Normale Supérieure de Lyon, 46 allée d'Italie, 69364 Lyon Cedex 07, tel: (33)-4-72-72-88-16, email: renaud.mahieux@ens-lyon.fr

\section{FINANCIAL DISCLOSURE}

RM was supported by INSERM and by a "contrat d'Interface vers l'hôpital" INSERM/AP-HP and is now supported by Ecole Normale Superieure de Lyon. FM and EW are supported by INSERM. PVA was supported by the Ministère de la Recherche and the Pasteur-Weizmann Association. We acknowledge the support from "La Ligue Nationale Contre le Cancer", l'Association de Recherche contre le Cancer (\#1085) and "Cent pour sang la vie". We also acknowledge the financial support of INSERM to our group. 


\section{REFERENCES}

1. Poiesz BJ, Ruscetti FW, Gazdar AF, Bunn PA, Minna JD, Gallo RC. Detection and isolation of type $\mathrm{C}$ retrovirus particles from fresh and cultured lymphocytes of a patient with cutaneous T-cell lymphoma. Proc Natl Acad Sci U S A. 1980;77(12):7415-7419.

2. Yoshida M, Miyoshi I, Hinuma Y. Isolation and characterization of retrovirus from cell lines of human adult T-cell leukemia and its implication in the disease. Proc Natl Acad Sci U S A. 1982;79(6):2031-2035.

3. Gessain A, Barin F, Vernant JC, et al. Antibodies to human T-lymphotropic virus type-I in patients with tropical spastic paraparesis. Lancet. 1985;2(8452):407-410.

4. Osame M, Usuku K, Izumo S, et al. HTLV-I associated myelopathy, a new clinical entity. Lancet. 1986;1(8488):1031-1032.

5. Rodgers-Johnson P, Gajdusek DC, Morgan OS, Zaninovic V, Sarin PS, Graham DS. HTLV-I and HTLV-III antibodies and tropical spastic paraparesis. Lancet. 1985;2(8466):1247-1248.

6. Bittencourt AL, Primo J, Oliveira MF. Manifestations of the human T-cell lymphotropic virus type I infection in childhood and adolescence. J Pediatr (Rio J). 2006;82(6):411-420.

7. Kira J, Fujihara K, Itoyama Y, Goto I, Hasuo K. Leukoencephalopathy in HTLV-Iassociated myelopathy/tropical spastic paraparesis: MRI analysis and a two year follow-up study after corticosteroid therapy. J Neurol Sci. 1991;106(1):41-49.

8. Lehky TJ, Levin MC, Kubota R, et al. Reduction in HTLV-I proviral load and spontaneous lymphoproliferation in HTLV-I-associated myelopathy/tropical spastic paraparesis patients treated with humanized anti-Tac. Ann Neurol. 1998;44(6):942-947.

9. Lezin A, Gillet N, Olindo S, et al. Histone deacetylase mediated transcriptional activation reduces proviral loads in HTLV-1 associated myelopathy/tropical spastic paraparesis patients. Blood. 2007;11 (10):3722-3728.

10. Taylor GP, Goon P, Furukawa Y, et al. Zidovudine plus lamivudine in Human TLymphotropic Virus type-I-associated myelopathy: a randomised trial. Retrovirology. 2006;3:63.

11. Bhigjee AI, Wiley CA, Wachsman W, et al. HTLV-I-associated myelopathy: clinicopathologic correlation with localization of provirus to spinal cord. Neurology. 1991;41(12):1990-1992.

12. Nagai M, Brennan MB, Sakai JA, Mora CA, Jacobson S. CD8(+) T cells are an in vivo reservoir for human T-cell lymphotropic virus type I. Blood. 2001;98(6):1858-1861.

13. Hanon E, Stinchcombe JC, Saito M, et al. Fratricide among CD8(+) T lymphocytes naturally infected with human T cell lymphotropic virus type I. Immunity. 2000;13(5):657664.

14. Afonso PV, Ozden S, Cumont MC, et al. Alteration of blood-brain barrier integrity by retroviral infection. PLoS Pathog. 2008;4(11):e1000205.

15. Ceccaldi PE, Delebecque F, Prevost MC, et al. DC-SIGN facilitates fusion of dendritic cells with human T-cell leukemia virus type 1-infected cells. J Virol. 2006;80(10):4771-4780.

16. Jones KS, Petrow-Sadowski C, Huang YK, Bertolette DC, Ruscetti FW. Cell-free HTLV-1 infects dendritic cells leading to transmission and transformation of CD4(+) T cells. Nat Med. 2008;14(4):429-436.

17. Olindo S, Lezin A, Cabre P, et al. HTLV-1 proviral load in peripheral blood mononuclear cells quantified in $100 \mathrm{HAM}$ /TSP patients: a marker of disease progression. $J$ Neurol Sci. 2005;237(1-2):53-59. 
18. Olindo S, Cabre P, Lezin A, et al. Natural history of human T-lymphotropic virus 1associated myelopathy: a 14-year follow-up study. Arch Neurol. 2006;63(11):1560-1566.

19. Nagai M, Usuku K, Matsumoto W, et al. Analysis of HTLV-I proviral load in 202 HAM/TSP patients and 243 asymptomatic HTLV-I carriers: high proviral load strongly predisposes to HAM/TSP. J Neurovirol. 1998;4(6):586-593.

20. Takenouchi N, Yamano Y, Usuku K, Osame M, Izumo S. Usefulness of proviral load measurement for monitoring of disease activity in individual patients with human $\mathrm{T}$ lymphotropic virus type I-associated myelopathy/tropical spastic paraparesis. J Neurovirol. 2003;9(1):29-35.

21. Matsuzaki T, Nakagawa M, Nagai M, et al. HTLV-I proviral load correlates with progression of motor disability in HAM/TSP: analysis of 239 HAM/TSP patients including 64 patients followed up for 10 years. J Neurovirol. 2001;7(3):228-234.

22. Jeffery KJ, Siddiqui AA, Bunce M, et al. The influence of HLA class I alleles and heterozygosity on the outcome of human $\mathrm{T}$ cell lymphotropic virus type I infection. $J$ Immunol. 2000;165(12):7278-7284.

23. Jeffery KJ, Usuku K, Hall SE, et al. HLA alleles determine human T-lymphotropic virus-I (HTLV-I) proviral load and the risk of HTLV-I-associated myelopathy. Proc Natl Acad Sci U S A. 1999;96(7):3848-3853.

24. Bangham CR. The immune control and cell-to-cell spread of human T-lymphotropic virus type 1. J Gen Virol. 2003;84(Pt 12):3177-3189.

25. Saito M, Nakagawa M, Kaseda S, et al. Decreased human T lymphotropic virus type I (HTLV-I) provirus load and alteration in T cell phenotype after interferon-alpha therapy for HTLV-I-associated myelopathy/tropical spastic paraparesis. J Infect Dis. 2004;189(1):29-40.

26. Mortreux F, Gabet AS, Wattel E. Molecular and cellular aspects of HTLV-1 associated leukemogenesis in vivo. Leukemia. 2003;17(1):26-38.

27. Taniguchi Y, Nosaka K, Yasunaga J, et al. Silencing of human T-cell leukemia virus type I gene transcription by epigenetic mechanisms. Retrovirology. 2005;2:64.

28. Ego T, Ariumi Y, Shimotohno K. The interaction of HTLV-1 Tax with HDAC1 negatively regulates the viral gene expression. Oncogene. 2002;21 (47):7241-7246.

29. Lu H, Pise-Masison CA, Linton R, et al. Tax relieves transcriptional repression by promoting histone deacetylase 1 release from the human T-cell leukemia virus type 1 long terminal repeat. J Virol. 2004;78(13):6735-6743.

30. Lagneaux L, Gillet N, Stamatopoulos B, et al. Valproic acid induces apoptosis in chronic lymphocytic leukemia cells through activation of the death receptor pathway and potentiates TRAIL response. Exp Hematol. 2007;35(10):1527-1537.

31. Nishioka C, Ikezoe T, Yang J, et al. Histone deacetylase inhibitors induce growth arrest and apoptosis of HTLV-1-infected T-cells via blockade of signaling by nuclear factor kappaB. Leuk Res. 2007; 32(2):287-296.

32. Lezin A, Olindo S, Belrose G, et al. Gene activation therapy: from the BLV model to HAM/TSP patients. Front Biosci (Schol Ed). 2009;1:205-215.

33. Toulza F, Nosaka K, Takiguchi M, et al. FoxP3(+) regulatory T cells are distinct from leukemia cells in HTLV-1-associated adult T-cell leukemia. Int $J$ Cancer. 2009;125(10):2375-2382.

34. Wolfe ND, Heneine W, Carr JK, et al. Emergence of unique primate T-lymphotropic viruses among central African bushmeat hunters. Proc Natl Acad Sci $U$ S A. 2005;102(22):7994-7999.

35. Allan JS, Leland M, Broussard S, Mone J, Hubbard G. Simian T-cell lymphotropic Viruses (STLVs) and lymphomas in African nonhuman primates. Cancer Invest. 2001;19(4):383-395. 
36. Gabet AS, Mortreux F, Talarmin A, et al. High circulating proviral load with oligoclonal expansion of HTLV-1 bearing T cells in HTLV-1 carriers with strongyloidiasis. Oncogene. 2000;19(43):4954-4960.

37. Monceaux V, Ho Tsong Fang R, Cumont MC, Hurtrel B, Estaquier J. Distinct cycling CD4(+)- and CD8(+)-T-cell profiles during the asymptomatic phase of simian immunodeficiency virus SIVmac251 infection in rhesus macaques. $J$ Virol. 2003;77(18):10047-10059.

38. Asquith B, Mosley AJ, Heaps A, et al. Quantification of the virus-host interaction in human T lymphotropic virus I infection. Retrovirology. 2005;2:75.

39. Lee B, Tanaka Y, Tozawa H. Monoclonal antibody defining tax protein of human Tcell leukemia virus type-I. Tohoku J Exp Med. 1989;157(1):1-11.

40. Nagai M, Kubota R, Greten TF, Schneck JP, Leist TP, Jacobson S. Increased activated human T cell lymphotropic virus type I (HTLV-I) Tax11-19-specific memory and effector CD8+ cells in patients with HTLV-I-associated myelopathy/tropical spastic paraparesis: correlation with HTLV-I provirus load. J Infect Dis. 2001;183(2):197-205.

41. Gout $\mathrm{O}$, Gessain A, Iba-Zizen $\mathrm{M}$, et al. The effect of zidovudine on chronic myelopathy associated with HTLV-1. J Neurol. 1991;238(2):108-109.

42. Sheremata WA, Benedict D, Squilacote DC, Sazant A, DeFreitas E. High-dose zidovudine induction in HTLV-I-associated myelopathy: safety and possible efficacy. Neurology. 1993;43(10):2125-2129.

43. Iwanaga M, Watanabe T, Utsunomiya A, et al. Human T-cell leukemia virus type I (HTLV-1) proviral load and disease progression in asymptomatic HTLV-1 carriers: a nationwide prospective study in Japan. Blood. 


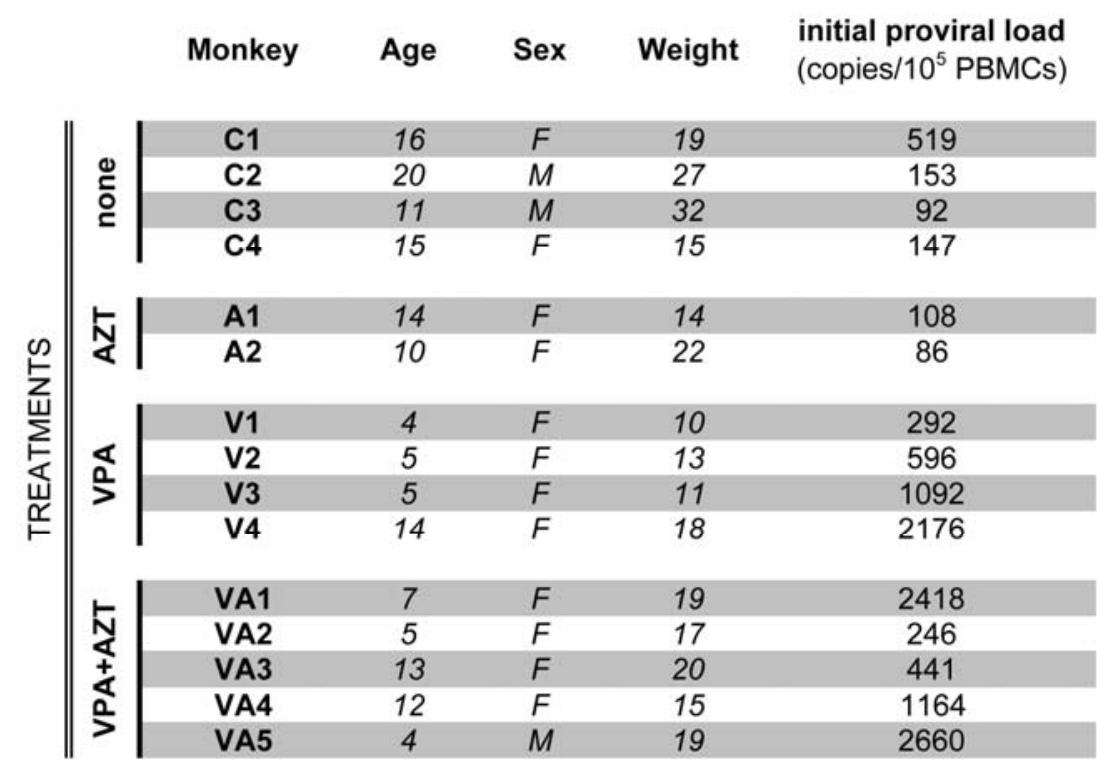

\section{Table 1: Experimental protocol.}

Fifteen STLV-1 infected Papio papio were given daily a dose of VPA and/or AZT per os. The STLV-1 proviral load values are presented as the Tax copy number per $10^{5}$ PBMCs at the initiation of the treatment. These values were also determined a month before the initiation of the trial and were found to be similar. 

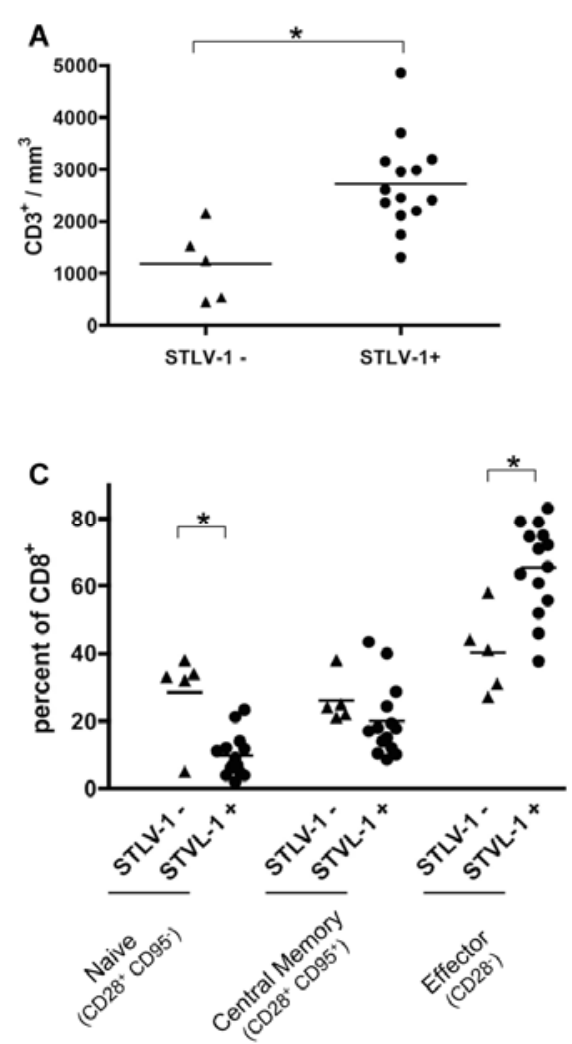
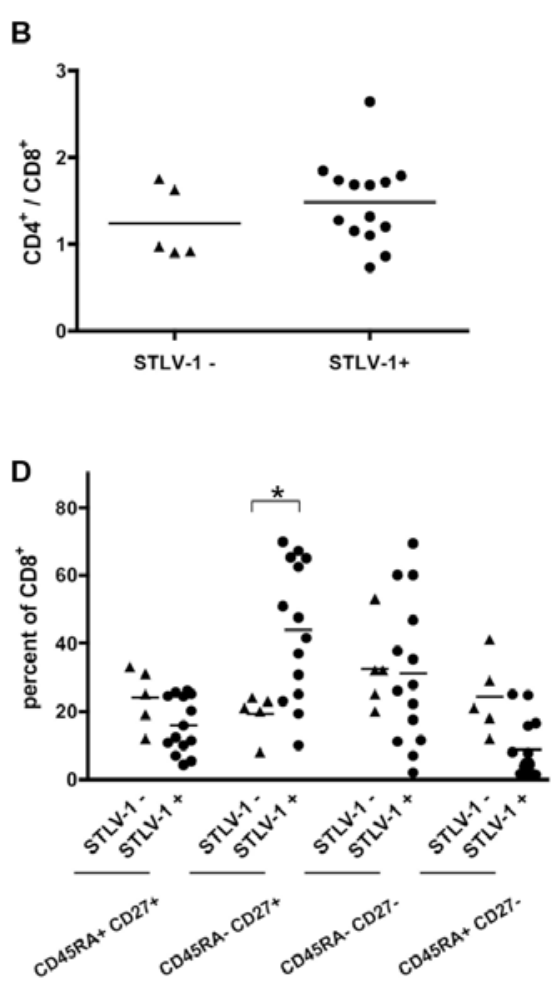

Figure 1: STLV-1 infection induces a constitutive activation of the simian immune system.

(A) Comparison of the $\mathrm{CD}^{+}$cell number per $\mathrm{mm}^{3}$ of blood between non-infected (5 animals) and STLV-1 infected (15 animals) monkeys before initiation of the treatment. Cells were stained with CD3 antibodies. The percentages obtained were then reported to the total lymphocyte number determined by clinical automated lymphocyte count.

(B) Comparison of $\mathrm{T}-\mathrm{CD} 4^{+} / \mathrm{T}-\mathrm{CD} 8^{+}$ratio between non-infected and infected monkeys before treatment. Cells were stained with CD4, CD8 and CD3 antibodies.

(C) Comparison of $\mathrm{CD}^{+}$subpopulations count per $\mathrm{mm}^{3}$ of blood determined before treatment. Cells were stained for CD3, CD8, CD95 and CD28.

Significant differences $(\mathrm{p}<0.05)$ under Mann-Whitney tests are outlined with the asterisk. 

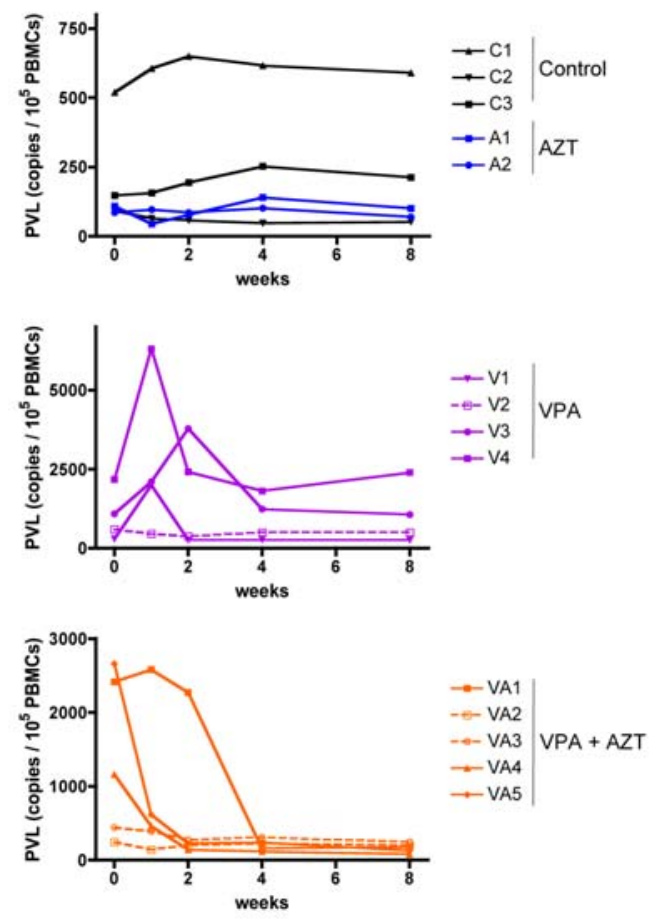

Figure 2: Combined VPA/AZT treatment is linked to a significant reduction of the PVL in STLV-1 asymptomatic carriers.

The PVL (copy number $/ 10^{5}$ PBMCs) was determined by real time PCR, as previously described ${ }^{36}$. For a given individual, all samples were run at the same time, to avoid interexperiment variation. The values presented here are the mean of two independent assays. $\mathrm{C}$ : controls; A: AZT, V: valproate; VA: Valproate + AZT 
A
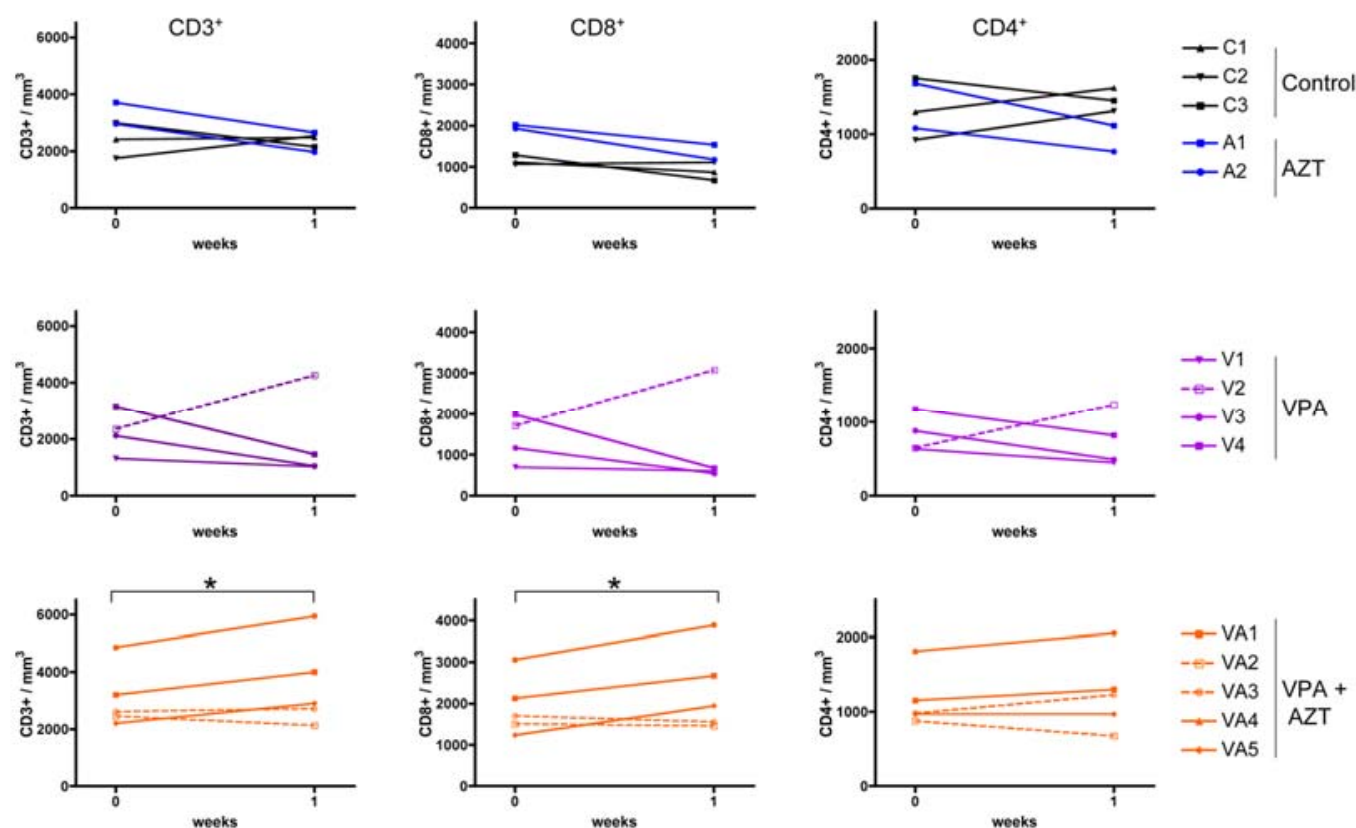

B
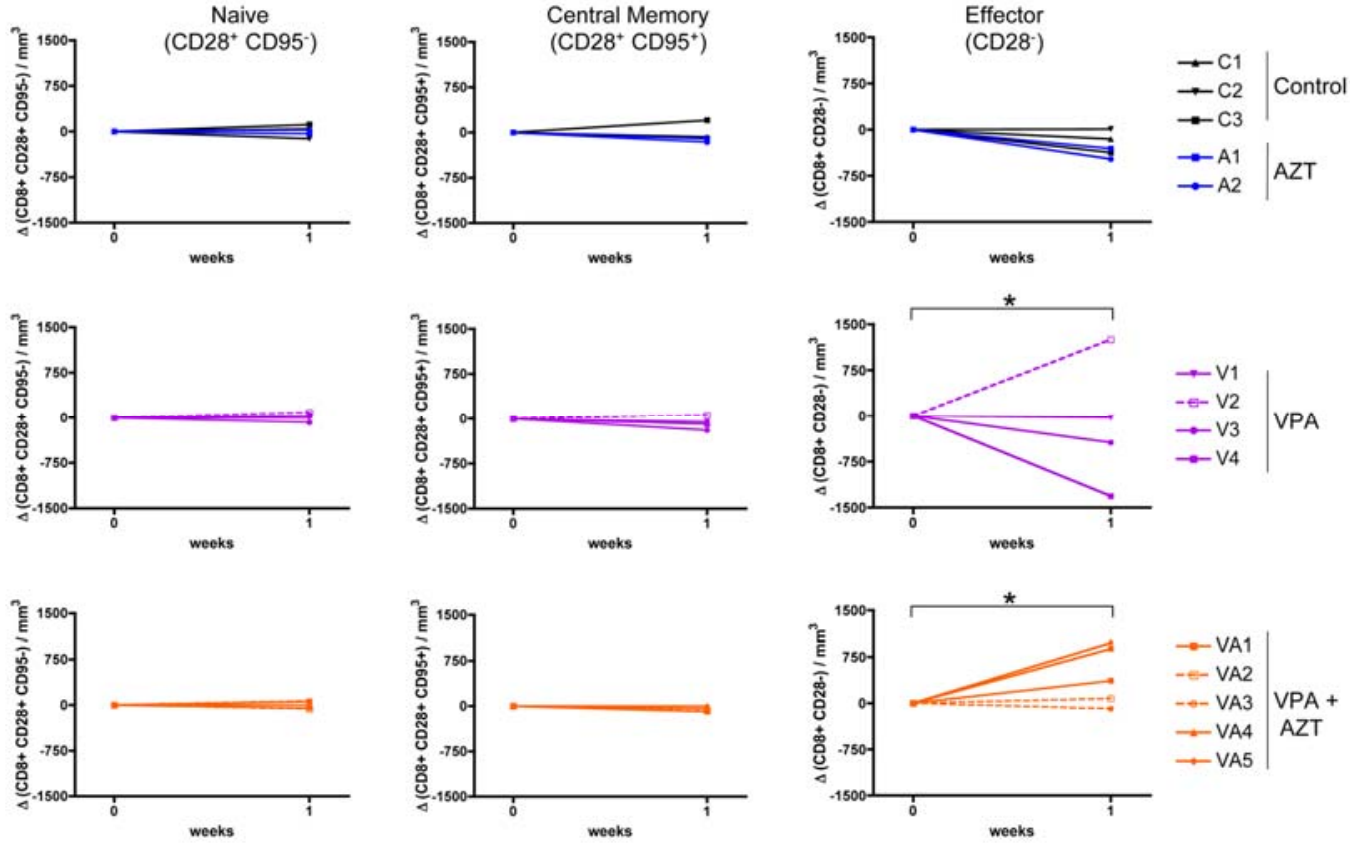

Figure 3: VPA/AZT treatment to an accumulation of $\mathrm{CD8}^{+}$effector memory $\mathrm{T}$ lymphocytes.

(A) Variation of $\mathrm{CD}^{+}, \mathrm{CD}^{+}$and $\mathrm{CD}^{+}$cell number per $\mathrm{mm}^{3}$ of blood was determined at day 0 and 7 of treatment. Cells were stained with CD4, CD8 and CD3 antibodies and analyzed by flow cytometry. The absolute numbers are derived from the absolute lymphocyte count previously determined by clinical automated systems.

(B) Variation in the $\mathrm{CD} 8^{+}$subpopulations count was determined at day 0 and 7 of treatment. Cells were stained for CD3, CD8, CD95 and CD28.

Significant differences $(\mathrm{p}<0.05)$ under paired-Student's t-tests are outlined with the asterisk. 

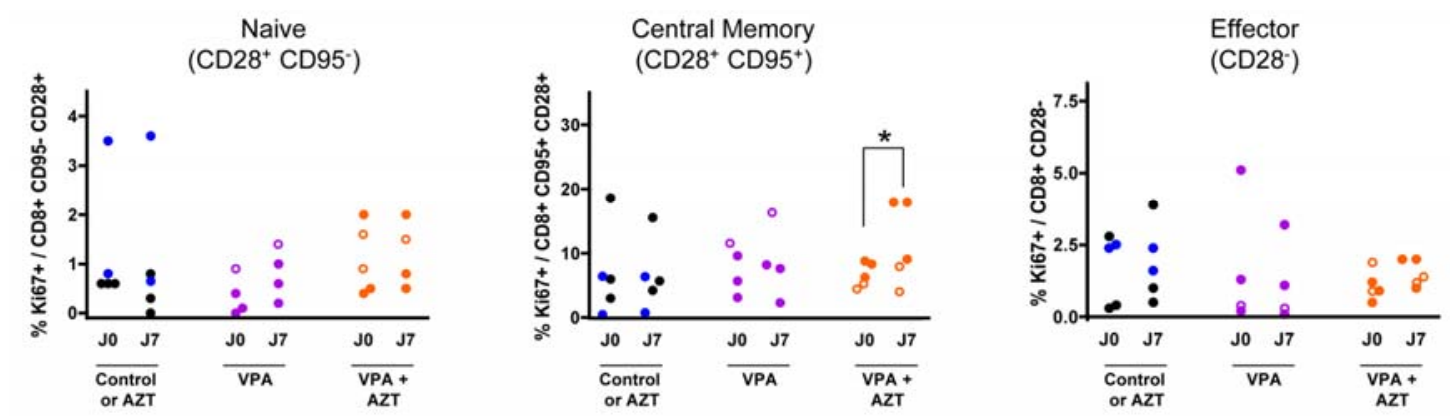

Figure 4: VPA/AZT treatment induces a proliferation of $\mathrm{CD8}^{+}$central memory $\mathrm{T}$ lymphocytes.

Variations in Ki67 expression (a marker for cell proliferation) was determined within the CD8 ${ }^{+}$T-cells subpopulations. The cells were stained for CD8, CD28, CD95 and Ki67.

Statistical differences $(\mathrm{p}<0.05)$ under paired-Student's t-tests are outlined with the asterisk.

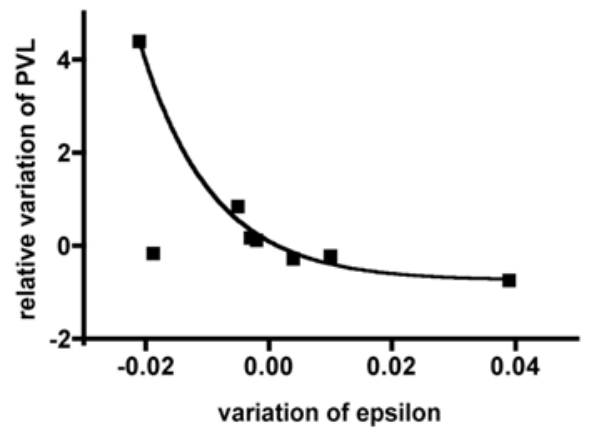

Figure 5: STLV-1 PVL evolution is inversely correlated with the rate of $\mathrm{CD8}^{+}$cellmediated lysis of STLV-1-infected cells.

The lytic efficiency rates (or epsilon) were determined at different times (at 0,1 and 2 weeks of treatment) for the 4 baboons, whose Tax-expression was detectable by flow cytometry in the PBMCs. The variations of the epsilon value over a time period of one week were plotted against the corresponding variations of PVL. The plot is overlaid with the line of best.

The variations of PVL and epsilon correlate significantly according to a one-phase exponential decay model $\left(R^{2}=0.63\right.$, or $R^{2}=0.989$ if the outlier point is excluded $)$. 